\title{
Terminologie et ontologie pour les humanités numériques : le cas des vêtements de la Grèce antique
}

Terminology and Ontology for Digital Humanities: The Case of Ancient Greek Dress

Christophe Roche et Maria Papadopoulou

\section{OpenEdition Journals}

Édition électronique

URL : https://journals.openedition.org/revuehn/462

DOI : $10.4000 /$ revuehn.462

ISSN : 2736-2337

\section{Éditeur}

Humanistica

\section{Référence électronique}

Christophe Roche et Maria Papadopoulou, « Terminologie et ontologie pour les humanités

numériques: le cas des vêtements de la Grèce antique », Humanités numériques [En ligne], 2 | 2020 mis en ligne le 01 juin 2020, consulté le 17 juillet 2021. URL : http://journals.openedition.org/revuehn/ 462 ; DOI : https://doi.org/10.4000/revuehn.462

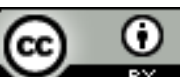

Les contenus de la revue Humanités numériques sont mis à disposition selon les termes de la Licence Creative Commons Attribution 4.0 International. 


\title{
humanités \\ numériques
}

\section{Terminologie et ontologie pour les humanités numériques : le cas des vêtements de la Grèce antique \\ Terminology and Ontology for Digital Humanities: The Case of Ancient Greek Dress}

\author{
Christophe Roche et Maria Papadopoulou
}

\section{Résumés}

Cet article présente les résultats d'une collaboration entre une philologue et un terminologue initiée lors d'un projet européen portant sur les vêtements de la Grèce antique (Marie Skłodowska Curie Actions Project Chlamys. The Cultural Biography of a Garment in Hellenistic Egypt, grant 657898).

Le flou terminologique qui règne autour des études consacrées aux vêtements de cette époque, dont les sources sont souvent lacunaires (Delaporte 1981, 7), entrave fortement la communication et le partage de connaissances entre experts. Identifier clairement ce que désignent les termes dans ce qu'ils ont de précis, mais aussi de vague, et également bien nommer les choses ont été au cœur de notre projet, qui a nécessité une représentation explicite des connaissances définissant les différents types de vêtements.

À cette préoccupation d'ordre terminologique s'est ajouté le besoin de pouvoir identifier un vêtement donné en fonction de ses caractéristiques, c'est-à-dire de pouvoir identifier (classifier) le ou les types de vêtements auxquels il appartient. Il convient de noter qu'aujourd'hui il n'existe pas de système de classification (semi-)automatisé des vêtements en tant qu'artéfacts culturels des cultures passées qui soit unanimement reconnu.

C'est la raison pour laquelle nous nous sommes intéressés à la notion d'ontologie issue de l'ingénierie des connaissances et du Web sémantique, et par conséquent à celle d'ontoterminologie, terminologie dont le système conceptuel est une ontologie formelle. Il a fallu alors définir une 
méthodologie de construction de l'ontoterminologie, faisant intervenir successivement les dimensions linguistique et conceptuelle. Celle-ci repose sur un ensemble de caractéristiques essentielles, connaissances " primitives " partagées par les experts, à partir desquelles se construit lontologie du domaine.

Le résultat est une terminologie multilingue (anglais, français, grec) dont la signification des termes repose sur une ontologie du domaine des vêtements de la Grèce antique, posée ici comme partagée et consensuelle, et qui peut donner lieu à de nombreuses applications informatiques : dictionnaires terminologiques multilingues, moteurs de recherche sémantiques multilingues, classification automatique, gestion documentaire, gestion des connaissances, etc.

Ce projet a mis en évidence l'importance, voire la nécessité, de disposer d'une méthode outillée reposant sur une approche pluridisciplinaire, conciliant les démarches " émique " et " étique ", et puisant à la linguistique, la terminologie et la modélisation des connaissances issue de l'intelligence artificielle ; une méthode qui permet la collaboration des différents intervenants et dont les résultats peuvent être partagés grâce à l'utilisation de formats d'échange respectant les standards en vigueur, dont ceux de l'ISO et du $\mathrm{W}_{3} \mathrm{C}$.

Cet article s'attachera plus particulièrement à présenter la méthode et l'outil employés dans le cadre de ce projet en ce qu'ils ont de généralisable pour des projets similaires relevant des humanités numériques, ainsi que certains des résultats obtenus.

This article presents the results of a collaboration between a classical philologist and a terminologist initiated during an EU-funded research project on ancient Greek clothing (Marie Skłodowska Curie Actions Project Chlamys. The Cultural Biography of a Garment in Hellenistic Egypt, grant 657898).

The terminological ambiguity prevailing in the studies on garments of this period, whose sources are often incomplete (Delaporte 1981, 7), severely hinders the communication and knowledge sharing among experts. Clearly identifying what the terms designate, in what is specific, but also vague, as well as the naming of garments was at the heart of our research, which demanded the explicit representation of the knowledge that defines the different types of clothing.

Added to this terminological concern is the need to enable the identification of a given garment according to its characteristics, i.e. to be able to identify (classify) the type or types of clothing to which it belongs. It should be noted that today there is no universally recognised (semi-)automated classification of garments as cultural artefacts of past cultures. This is why we are interested in the notion of ontology resulting from knowledge engineering and the semantic Web, and consequently in ontoterminology, a terminology whose conceptual system is a formal ontology. It was then necessary to define a methodology for the construction of such an ontoterminology, alternating the linguistic and conceptual dimensions. Ontoterminology defines concepts based on a set of essential characteristics, "knowledge primitives" shared by the experts, from which the ontology of the domain is built.

The result is a multilingual terminology whose meaning is based on an ontology of the clothing domain of ancient Greece, which is seen here as shared and consensual, and which can give rise to many computer ap- 
plications: multilingual terminology dictionaries, multilingual semantic search engines, automatic classification, document management, knowledge management, etc.

This project highlighted the importance, even the necessity, of having a tool-assisted method based on a multidisciplinary approach, reconciling "emic" and "etic" approaches, and drawing on linguistics, terminology and knowledge modelling, a field of artificial intelligence. It also highlighted the importance of adopting a method that allows the collaboration of distributed end users, and whose results can be shared through the use of exchange formats complying with the standards in force, including those of the ISO and of the $\mathrm{W}_{3} \mathrm{C}$.

This article will focus on presenting the method and tool used in this project as they have generalisable features for similar projects in digital humanities, as well as some of the results obtained.

\section{Entrées d'index}

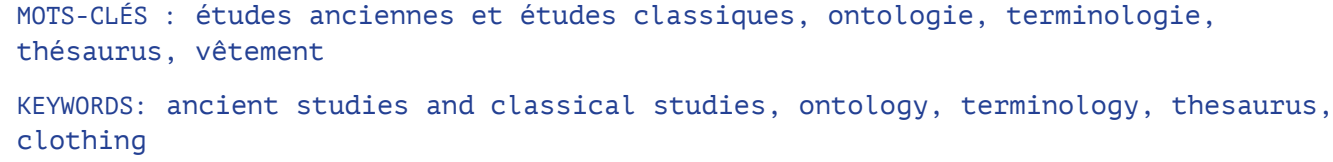

Studies of garment-terms in historical societies tend to be hampered by a lack of understanding of the specific vocabulary of dress. Llewellyn-Jones, L. (2003)

\section{Problématique}

Omniprésent dans la vie quotidienne et partie intégrante de l'espace culturel, le vêtement ${ }^{1}$ est un sujet d'étude dont l'importance n'est plus à montrer ; que ce soit, par exemple, comme marqueur social (classe sociale, fonction au sein de la cité ${ }^{2}$, genre) ou pour lui-même en tant qu'élément représentatif des matériaux, techniques et savoir-faire d'une époque donnée. Il constitue donc un support privilégié pour l'étude de la Grèce antique.

Malheureusement, fabriqués à partir de matériaux hautement biodégradables, le peu de vêtements qui nous sont parvenus est souvent dans un état tel qu'il est extrêmement difficile de les reconnaître. Les principales sources d'information, souvent lacunaires et inégalement réparties selon les époques (certaines étant surreprésentées, d'autres sousreprésentées), sont soit textuelles (textes primaires : inscriptions, ostraca, papyrus), soit visuelles à travers la représentation de vêtements sur différents supports : vases, statues, bas-reliefs, pièces de monnaie, etc. Le problème est alors celui de l'appariement des mots avec les objets, que ces objets soient contenus dans le registre archéologique ou figurent dans les sources visuelles et textuelles. Y répondre peut considérablement améliorer nos connaissances et notre compréhension de cette culture. 
Idéalement, les termes que nous recherchons peuvent être trouvés dans les textes grecs anciens. Cependant, faire correspondre les objets à leur nom n'est pas simple pour la culture matérielle grecque en général ${ }^{3}$, car les " preuves " provenant des sources textuelles et archéologiques ne sont pas coextensives. En effet, il existe des vêtements clairement identifiés sur des sources visuelles dont le nom ancien n'est pas connu, tout comme il y a des termes dans les textes en grec ancien dont la référence (vêtement désigné par le terme) demeure inconnue. De plus, certains termes grecs anciens sont polysémiques au sens où ils peuvent désigner différents types de vêtements. À cela s'ajoute le fait que certains termes, bien ancrés dans l'usage, désignant des vêtements de la Grèce antique ont été créés à l'époque moderne (post-Renaissance) même s'ils donnent l'impression d'être du grec ancien principalement en raison de leur morphologie. Bien que justifié au regard des connaissances du domaine acquises à un moment donné, le fait de nommer les choses avec des termes à consonance antique sans référence croisée avec les sources textuelles a été la source d'une confusion considérable. Il en résulte un flou terminologique ${ }^{4}$ qui nuit grandement aux études classiques, à la communication et au partage des connaissances entre les individus, qu'ils soient philologues, archéologues ou simples utilisateurs : " le flou terminologique [...] dans les études consacrées au costume [...] peut être une cause de difficultés de communication entre chercheurs : flou dans les descriptions des pièces, avec abus de termes partout dans le monde, comme poncho ou sari, mais aussi imprécision en ce qui concerne les termes les plus généraux, costume, vêtement, habillement, que chaque auteur connote différemment" (Delaporte 1981, 12). Et Balfet et al. (1984) d'ajouter « un babélisme terminologique qui entrave la communication entre chercheurs".

Il ne peut donc y avoir communication, et a fortiori échange et partage de connaissances, sans s'accorder au préalable sur la signification (définition) des termes échangés, c'est-à-dire sur la définition des différents types de vêtements, sachant que la typologie utilisée par les spécialistes d'aujourd'hui n'est probablement pas identique à celle utilisée dans l'Antiquité : "Bien que la terminologie grecque et latine utilisée par les spécialistes pour décrire les vêtements anciens ne soit pas celle qui était utilisée dans l'Antiquité pour désigner des vêtements particuliers, c'est un vocabulaire utile de l'habillement " ${ }^{5}$ (Stears 2006, 226). "Pour décrire un segment de la culture antique il est inévitable que nous usions de mots et de catégories appartenant à notre expérience culturelle ", remarque Bettini $(2012,173)$ à propos de son analyse d'une divinité romaine. Et il continue en citant Clifford Geertz : " Naturellement, les anthropologues du monde antique ne peuvent espérer fournir des interprétations entièrement construites en utilisant des catégories locales probablement ils ne doivent pas même chercher à le faire. Selon l'avis de Geertz, la tâche du chercheur est la suivante : prendre des concepts proches de l'expérience de la population étudiée et "les placer dans une connexion éclairante" avec les "concepts que les théoriciens ont façonnés pour capturer les traits généraux de la vie sociale” ; ceci à fin de "produire une interprétation des façons de vivre propres à une certaine population qui ne soit ni emprisonnée dans l'horizon mental de cette population - une ethnographie de la sorcellerie écrite par une sorcière ni systématiquement sourde aux tonalités distinctives de son existence - 
une ethnographie de la sorcellerie écrite par un géomètre. " L'identification et la définition des types de vêtements proposés dans le cadre de nos travaux visent à concilier les approches "émique " et "étique " ${ }^{6}$, c'est-à-dire à concilier la façon dont les Grecs de l'Antiquité percevaient et organisaient le réel et la façon dont aujourd'hui nous l'analysons au regard de principes généraux. Concrètement, cela a consisté à rechercher, à travers l'étude des ressources linguistiques, matérielles et visuelles qui nous sont parvenues, les caractéristiques descriptives et définitoires des vêtements telles que les Grecs pouvaient les concevoir et à les organiser en axes d'analyse afin de proposer une conceptualisation des vêtements répondant à des critères épistémologiques et logiques posés comme " universels ${ }^{7}$ " (notions de caractéristique, de classification, etc.).

À cette préoccupation d'ordre terminologique s'est ajouté le besoin de pouvoir identifier un vêtement donné en fonction de ses caractéristiques, c'est-à-dire de pouvoir identifier (classifier) le ou les types de vêtements auxquels il appartient. Les chercheurs ont proposé différents systèmes de classification non automatisés pour les vêtements (Balfet et al. 1984 ; Eicher et al. 1992, 18, tableau 1.1 ; Buck 1982). Balfet et al. (1984) ont tenté de résoudre le problème en proposant une classification dans le but de " répondre au besoin d'un système de dénomination transculturelle des pièces vestimentaires ". Il convient de noter qu'aujourd'hui il n'existe pas de système de classification (semi-)automatisé des vêtements en tant qu'artéfacts culturels des cultures passées qui soit unanimement reconnu.

C'est la raison pour laquelle nous nous sommes intéressés à la notion d'ontologie ${ }^{8}$ issue de l'ingénierie des connaissances et du Web sémantique, et par conséquent à celle d'ontoterminologie, terminologie dont le système conceptuel est une ontologie formelle. Il a fallu alors définir une méthodologie de construction de l'ontoterminologie, alternant successivement les dimensions linguistique et conceptuelle, tout en proposant un « socle » de connaissances partagées sur lequel il est possible aux experts de s'accorder tout en préservant la diversité langagière. Le résultat est une terminologie multilingue (anglais, grec, français) dont la signification des termes repose sur une ontologie du domaine des vêtements de la Grèce antique et qui peut donner lieu à de nombreuses applications informatiques (Staab et Studer 2009) : dictionnaires terminologiques (figure 9), moteurs de recherche sémantiques et multilingues, classification automatique, gestion documentaire (export en SKOS), gestion des connaissances, etc.

Ce projet a mis en évidence l'importance, voire la nécessité, de disposer d'une méthode outillée reposant sur une approche pluridisciplinaire, conciliant les démarches "émique " et «étique ", puisant à la linguistique, la terminologie et la modélisation des connaissances permettant la collaboration des différents intervenants et dont les résultats peuvent être partagés grâce à l'utilisation de formats d'échange respectant les standards en vigueur dont ceux de l'ISO et du W3C (RDF/OWL, SKOS, HTML, CSV, JSON, Ontolex-Lemon, TEI-Lexo).

Dans la suite de l'article, on s'attachera plus particulièrement à présenter la méthode et l'outil employés dans le cadre de ce projet en ce qu'ils ont de généralisable pour des projets similaires relevant des humanités numériques, ainsi que certains des résultats obtenus. Nous 
commencerons par présenter les ressources utilisées pour la construction de l'ontoterminologie multilingue des vêtements de la Grèce antique. Suivra ensuite un chapitre dédié à la notion d'ontoterminologie, après avoir rappelé ce qu'on entend par terminologie et ontologie. Le quatrième chapitre sera consacré à l'outil Tedi, un éditeur d'ontoterminologies multilingues. Enfin nous présenterons la méthodologie mise en œuvre dans le cadre de notre projet, illustrée avec l'exemple de la chlamyde, méthode qui tient compte des retours d'expériences menées dans le cadre de deux projets européens sur le patrimoine culturel (Linked Heritage ${ }^{9}$ et AthenaPlus ${ }^{10}$ ).

Notons pour terminer ce premier chapitre que les vêtements de la Grèce antique ne sont pas les seuls objets matériels confrontés aux problèmes que nous venons d'énoncer. Comme le note avec justesse Martin Doerr (2009, 476), " malgré le fait que la classification automatisée est une discipline établie de longue date en archéologie, il n'existe aucun système terminologique largement accepté ${ }^{11}$ ". Notre approche, tant théorique que pratique, se veut applicable à d'autres domaines. Une étude est en cours sur la poterie de la Grèce antique.

\section{Ressources utilisées}

Ce chapitre présente brièvement les ressources utilisées dans le cadre de notre projet. Nous les avons structurées en quatre grandes classes : le corpus de référence, les dictionnaires de grec ancien, les collections des musées, et ce que nous avons regroupé sous l'expression de "métadonnées pour les humanités numériques ", qui comprend vocabulaires, terminologies, thésaurus et ontologies au sens où les sciences de l'information l'entendent. Précisons que notre objectif n'est pas ici l'extraction de termes à partir de corpus, ils sont connus des experts, ni de déterminer leurs différents sens en usage discursif, mais seulement de définir les termes au regard des connaissances qu'ils dénotent en dehors de leurs usages. La dimension diachronique n'est également pas prise en compte. On s'intéresse ici à la période correspondant aux époques classique et hellénistique, période pendant laquelle les connaissances portant sur les vêtements de la Grèce antique sont jugées suffisamment stables.

Le corpus de référence a été constitué à partir de textes accessibles en ligne provenant principalement des bibliothèques numériques Perseus 4.o de l'université Tufts ${ }^{12}$ et Thesaurus Linguae Graecae (TLG) en ligne de l'université de Californie à Irvine ${ }^{13}$.

Parmi les dictionnaires consultés citons, pour le grec ancien, le dictionnaire standard Greek-English Lexicon de Liddell, Scott et Jones (1940) - dénommé le $L S J$ - accessible en ligne à partir des deux sites précédemment cités (figure 1 avec " chlamyde " comme entrée) ; le dictionnaire encyclopédique de William Smith accessible à partir de Perseus 4.o, ainsi que le Dictionnaire des antiquités grecques et romaines (DAGR) édité par Charles-Victor Daremberg et Edmond Saglio, également accessible en ligne $^{14}$. C'est aussi le dictionnaire du Centre de recherche sur l'art classique (Classical Art Research Centre ${ }^{15}$ ), accessible en ligne, qui repose sur les archives Beazley contenant une des plus grandes collections d'images de poteries anciennes peintes. Ce dernier type de dictionnaires est doublement intéressant pour notre problématique. En effet, ces dic- 
tionnaires contiennent des définitions de choses, c'est-à-dire des descriptions des vêtements désignés par les termes, auxquelles sont associées des représentations de ces mêmes vêtements (peintures sur poteries, statues, monnaies, etc.) ${ }^{16}$.

FIGURE 1. DÉFINITION DE LA CHLAMYDE DANS LE LSJ

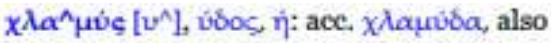

A. “ $\chi \lambda$ גanuv $v^{\circ}$ Sapph.674;-short mantle, worn prop. by horsemen, X.An.7-4.4; borrowed with the $\pi \varepsilon \dot{r a \sigma o s}$ from Thessaly, Philem.34, Poll.10.124; but said to be Macedonian, Arist.Fr.5oo,

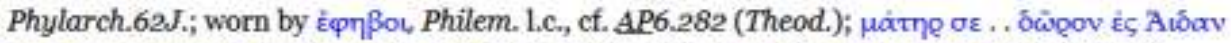

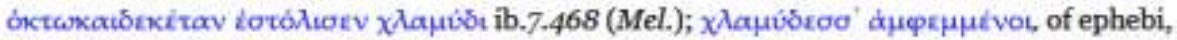

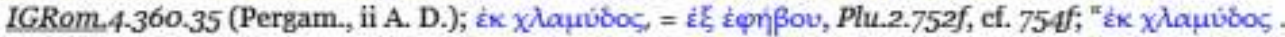

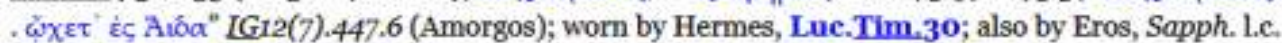
(v. Poll.10.124), Philostr.Im.1.6, ef. AP12.78 (Mel.).

Source : http://www.perseus.tufts.edu/hopper/text?

doc $=$ Perseus\%3Atext\%3A1999.04.0057\%3Aentry\%3Dxlamu\%2Fs

Les collections des musées, à travers la représentation des vêtements de la Grèce antique sur différents supports, peintures, peintures sur vases, sculptures, bas-reliefs, pièces de monnaie, constituent une source importante d'informations. Citons les sites de Harvard Art Museums ${ }^{17}$, de Arachne ${ }^{18}$ donnant accès à la base de données centrale des objets du German Archaeological Institute (DAI) et du Archaeological Institute de l'université de Cologne, le Metropolitan Museum of Art de New York ${ }^{19}$, et bien sûr celui d'Europeana ${ }^{20}$ donnant accès aux données des institutions culturelles européennes.

Nous avons regroupé sous l'expression de "métadonnées pour les humanités numériques " les vocabulaires, terminologies, thésaurus et ontologies au sens où la gestion de l'information l'entend ${ }^{21}$ et qui sont utilisés principalement pour la recherche d'information ${ }^{22}$. Si à strictement parler, les thésaurus ${ }^{23}$ ne sont pas des terminologies ni des ontologies ${ }^{24}$ (et réciproquement, Roche et al. 2014) - ils n'ont pas les mêmes finalités -, ils fournissent néanmoins de nombreuses informations utiles à travers les classifications qu'ils proposent et les définitions qui accompagnent parfois les termes (sous la forme de notes par exemple). Nous nous sommes particulièrement intéressés au thésaurus AAT (Art and Architecture Thesaurus) de la fondation Getty ${ }^{25}$, qui comporte une dimension multilingue. La figure 2 présente les informations retournées par la consultation en ligne de AAT pour la chlamyde, incluant une définition (note), les équivalents dans différentes langues et la position hiérarchique du concept associé. 


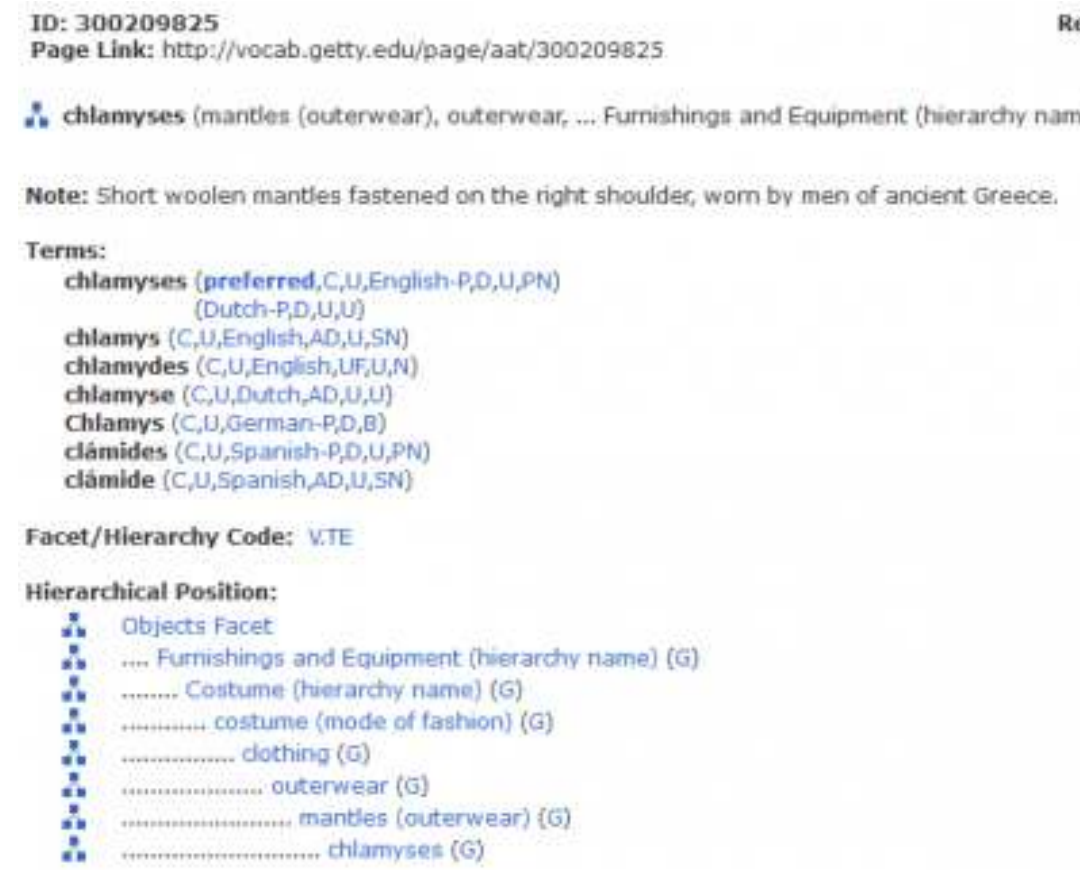

Source : http://vocab.getty.edu/page/aat/300209825

\section{Ontoterminologie}

Au même mot de "terminologie " se rattachent de multiples acceptions. Nous adopterons celles de la norme internationale ISO qui définit la terminologie à la fois comme une "science studying the structure, formation, development, usage and management of terminologies in various subject fields » (ISO 1087-1) et comme le résultat de son application à un domaine de spécialité : "set of designations belonging to one special language » où le terme est défini comme une "verbal designation of a general concept in a specific subject field» et le concept comme une « unit of knowledge created by a unique combination of characteristics».

Une terminologie a donc une double dimension, une dimension linguistique et une dimension conceptuelle, qui sont fortement liées puisque la définition du terme se calque sur celle du concept dénoté par le terme (on parle alors de définition de chose) ${ }^{26}$. La dimension conceptuelle représente les connaissances du domaine sous la forme d'un système de concepts liés par des relations (hiérarchiques, partitives, associatives) - ici les différents types de vêtements de la Grèce antique -, tandis que la dimension linguistique regroupe les différents termes désignant ces connaissances : " chlamys » en anglais, « chlamyde » en français, « $\chi \lambda \alpha \mu u \dot{s} \varsigma$ " en grec ancien et moderne, etc.

La modélisation du système conceptuel d'une terminologie sous la forme d'une ontologie ${ }^{27}$ au sens de l'ingénierie des connaissances (Gruber 1993 ; Uschold et Gruninger 1996 ; Guarino et al. 2009) constitue une des perspectives les plus prometteuses pour la terminologie (Roche 2005 ; Papadopoulou et Roche 2018). Elle conduit à la notion d'ontoterminologie (Roche 2012), terminologie dont le système conceptuel est une ontologie formelle. Expliciter le concept à l'aide d'un langage formel permet de garantir un certain nombre de propriétés telles que la cohé- 
rence (au sens de la théorie du concept sous-jacente), de consensus et de partage, si on considère que les concepts sont des connaissances extralinguistiques ${ }^{28}$ communes à une communauté de pratiques, mais aussi permet l'opérationnalisation des terminologies à des fins de traitement de l'information comme dans les moteurs de recherche sémantiques multilingues. Enfin, soulignons que l'ontoterminologie permet de normaliser la seule chose qui peut l'être, à savoir les connaissances du domaine, et de préserver ce qui doit l'être, à savoir la diversité linguistique.

\section{Environnement Tedi}

La construction de terminologies et d'ontologies est une tâche difficile qui requiert des outils spécifiques. Protégé ${ }^{29}$, éditeur libre d'ontologies supporté par une forte communauté d'utilisateurs, est un des plus connus. Basé sur les logiques de description (Baader et al. 2003) et supportant $\mathrm{OWL}^{30}$, le langage du $\mathrm{W}_{3} \mathrm{C}$ pour les ontologies, Protégé est un outil puissant de construction d'ontologies formelles. Cependant, sa maîtrise reste difficile pour des experts du domaine qui ne sont pas rompus à l'utilisation d'un langage formel comme la logique pour la définition de concepts : "As the group that developed Protégé, the most widely used ontology editor, we are keenly aware of how difficult the users perceive this task to be " (Horridge et al. 2013). C'est la raison pour laquelle nous nous sommes tournés vers l'environnement Tedi développé par l'université Savoie-Mont-Blanc. Tedi, ontoterminology editor ${ }^{31}$, est un environnement logiciel dédié à la construction d'ontoterminologies multilingues partageant une conceptualisation commune ${ }^{32}$ modélisée sous la forme d'une ontologie formelle. Contrairement à Protégé, Tedi n’a pas une visée universelle. Il repose sur une théorie du concept dédiée à la terminologie et intègre des principes épistémologiques qui se veulent proches du mode de pensée des experts.

\section{Présentation}

Tedi est composé d'un ensemble d'éditeurs (figure 3) dédiés à la dimension conceptuelle (éditeurs de concepts, d'objets, d'axes d'analyse, d'attributs, de relations) et à la dimension linguistique (éditeurs de termes et de noms propres). Il propose également différentes fonctionnalités liées à la recherche, à l'analyse des ontoterminologies et à l'export des données. Ces éditeurs et fonctionnalités reposent sur une théorie du concept dont Tedi exploite les principes afin de proposer des guides méthodologiques et de vérifier la cohérence du système lors de la construction des ontoterminologies. Nous ne présenterons ici que l'éditeur de concepts et l'éditeur de termes ainsi qu'une partie des fonctionnalités de Tedi (fonction de recherche, génération " automatique " de dénominations de concept, de patterns de définition pour les termes, etc.). 


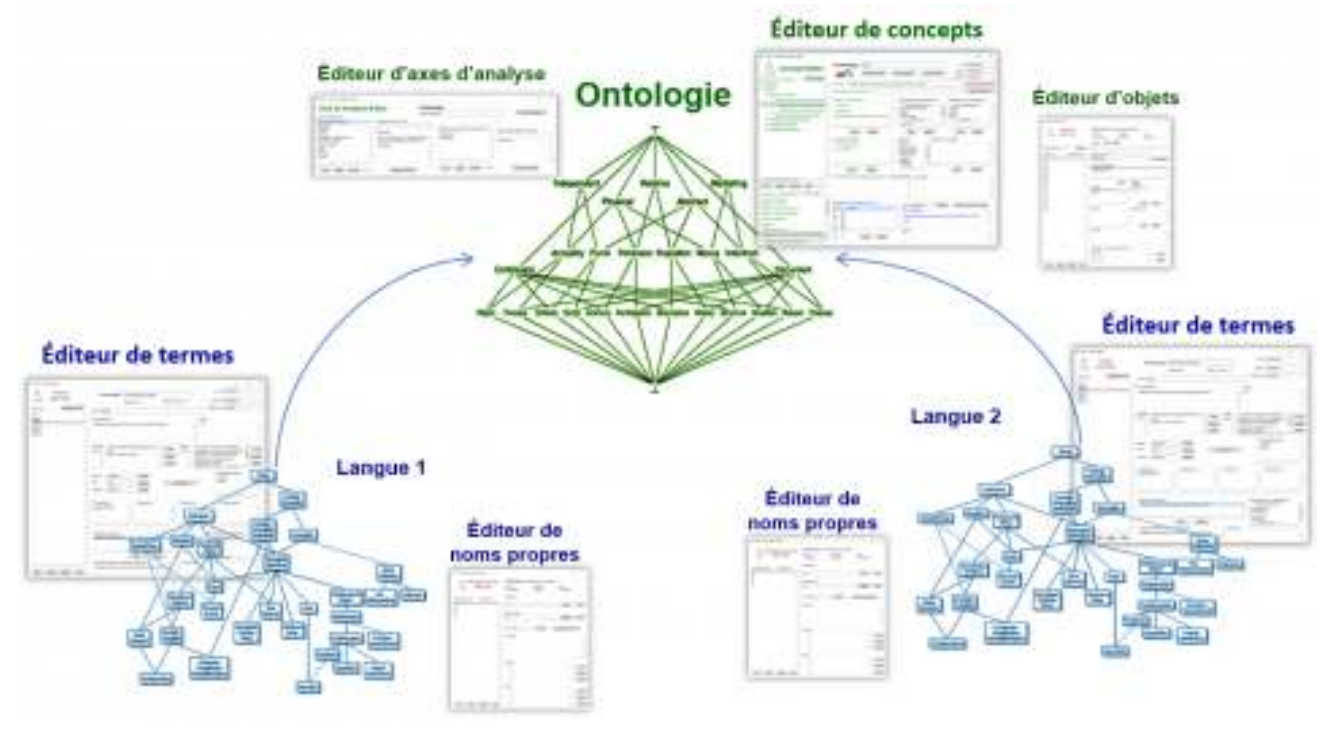

Ontoterminologies multilingues partageant une même conceptualisation

Notons enfin que Tedi permet à différents utilisateurs de gérer leurs propres terminologies tout en partageant une même ontologie autorisant ainsi la comparaison des différentes terminologies au regard du système conceptuel.

\section{Théorie du concept}

Tedi repose sur une théorie du concept proche de celle de l'ISO et du mode de raisonnement des experts selon laquelle un concept est défini comme une combinaison unique de caractéristiques. Cette théorie distingue les notions de caractéristique essentielle ${ }^{33}$ (notée " différence " dans Tedi) comme /with sleeves/ ou /attached $/{ }^{34}$ et de caractéristique descriptive $^{35}$ (appelée " attribut " dans Tedi) comme la couleur ${ }^{36}$ ou la nature de la fibre qui compose le vêtement. Les caractéristiques essentielles sont structurées en axes d'analyse à partir desquels se définissent les concepts. Les caractéristiques essentielles au sein d'un même axe d'analyse sont exclusives les unes des autres, par exemple l'axe d'analyse «body part " est défini par les caractéristiques /around body/, /around head/, /around waist/ et /leg encasing/. Les caractéristiques essentielles peuvent être dépendantes les unes des autres, ainsi le nombre d'attaches n'a de sens que si le vêtement se porte attaché et non enroulé autour du corps.

Les concepts se structurent en un système de concepts liés entre eux par différentes relations telles que la relation de subordination (générique $v s$ spécifique noté " isa " dans Tedi), la relation partitive ou toute autre relation associative que l'utilisateur peut définir ${ }^{37}$ en fonction de ses besoins. 


\section{Éditeur de concepts}

La définition des concepts et leur mise en relation se font à l'aide de l'éditeur de concepts (figure 4) qui s'appuie sur les principes épistémologiques et les propriétés logiques de la théorie du concept adoptée par Tedi pour guider l'utilisateur dans sa construction. Ainsi, seules les caractéristiques essentielles valides au sens de la théorie sont proposées à l'expert lors de la définition d'un concept. L'héritage par la relation générique des caractéristiques (essentielles et descriptives) et des relations est pris en compte et les informations héritées, mises à jour à chaque modification de la hiérarchie. Cette dernière peut être multiple, Tedi vérifiant la compatibilité des caractéristiques héritées. La définition des axes d'analyse, des caractéristiques essentielles (différences), descriptives (attributs) et des relations se fait également par l'intermédiaire d'éditeurs spécifiques accessibles via l'éditeur de concepts. À noter enfin, une fonction permet de rechercher de manière optimale un concept sur la sélection de caractéristiques essentielles ${ }^{38}$.

FIGURE 4. L'ÉDITEUR DE CONCEPTS DE TEDI

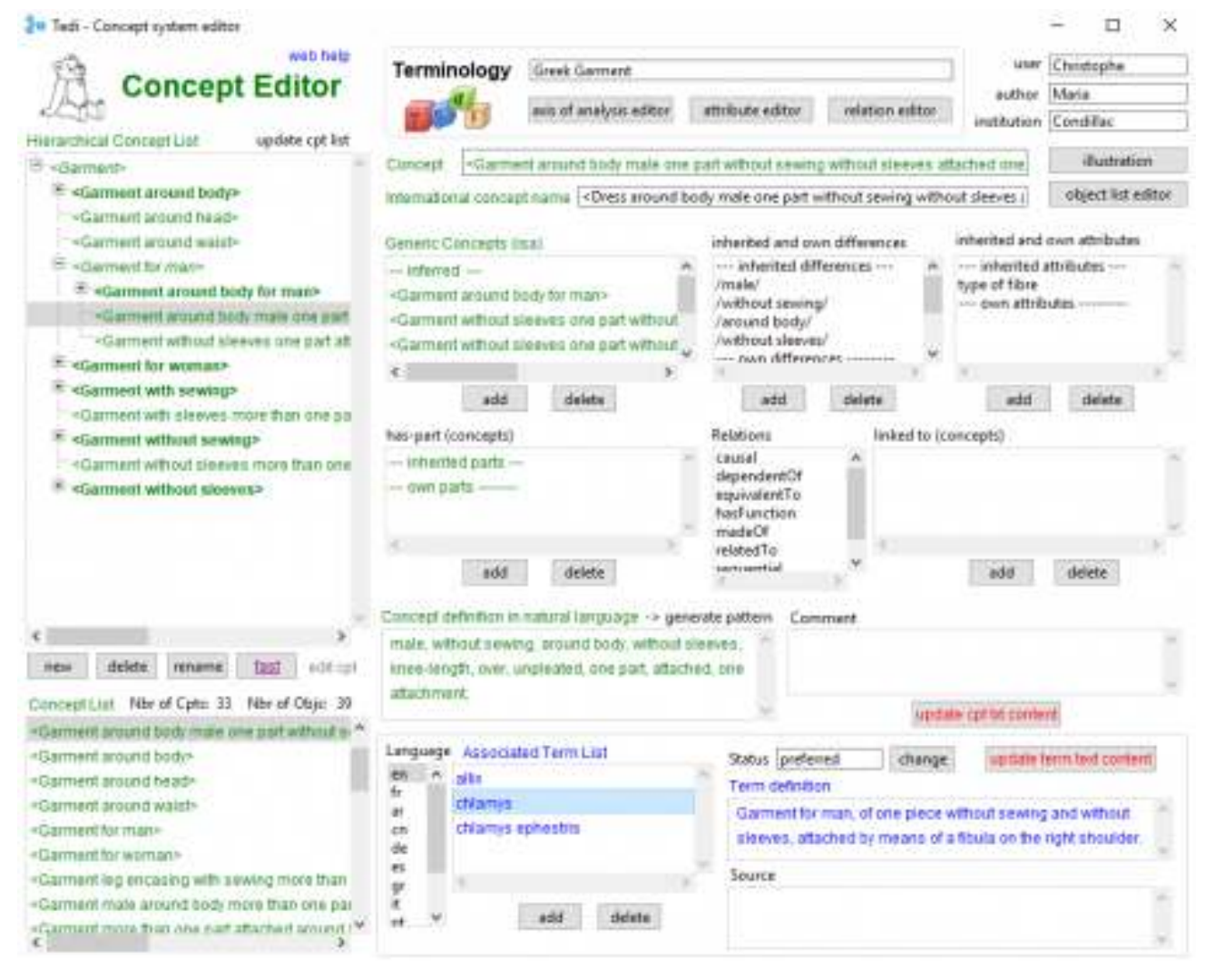

Capture d'écran du logiciel Tedi

\section{Éditeur de termes}

L'éditeur de termes de Tedi (figure 7) permet de saisir la définition d'un terme (un pattern de définition est proposé sur la base de la définition formelle du concept désigné par le terme) et un certain nombre d'informations associées telles que contextes, notes, statut (préféré, alternatif, toléré, déconseillé, obsolète), la catégorie grammaticale, les variations terminologiques et les formes fléchies. 
Notons que Tedi calcule automatiquement les équivalents terminologiques pour les différentes langues ainsi que les synonymes, hyperonymes et hyponymes terminologiques ${ }^{39}$ (figures 7 et 9).

\section{Formats d'échange}

Les ontoterminologies peuvent être exportées sous différents formats d'échange dont HTML, CSV, RDF/OWL, JSON. Les formats SKOS, Ontolex-Lemon et TEI-Lexo sont en cours de prise en compte.

\section{Méthodologie}

Il existe différentes méthodes de construction de terminologies et d'ontologies (citons pour ces dernières Uschold et King 1995 ; Grüninger et Fox 1995 ; Corcho et al. 2003 ; Buitelaar et al. 2005 ; Suárez-Figueroa, 2015). Le choix dépend de nombreux critères : l'objectif visé et sa finalité (une terminologie pour quoi faire ?), le type de terminologie (descriptive ou prescriptive), son éventuelle opérationnalisation (à des fins de traitement de l'information par exemple), le domaine d'application, les ressources mises à disposition (nature, format), la disponibilité des experts, etc. Ainsi on ne procède pas de la même façon si l'on doit produire un lexique de termes en usage dans un domaine touristique ou une terminologie normative dans le domaine de l'énergie nucléaire.

Le choix de donner la priorité au terme ou au concept n'est également pas sans conséquence sur la démarche et le résultat obtenu. Ce choix est parfois conditionné par l'indisponibilité des experts qui oblige à se reporter sur les autres ressources disponibles, en particulier textuelles. Si l'utilisation d'outils de traitement automatique de corpus apporte de nombreuses informations précieuses, il faut se garder de confondre les connaissances du domaine avec les discours auxquelles elles peuvent donner lieu. Les modèles " conceptuels " extraits à partir de textes ${ }^{40}$ se superposent rarement aux modèles conceptuels construits directement par les experts dans un langage dédié à l'expression de leurs connaissances : "le lexique des langues ne reflète pas la conception scientifique du monde » (Rastier 2004), « dire n'est pas concevoir » (Roche 2007).

La méthodologie que nous avons suivie pour la construction de l'ontoterminologie multilingue des vêtements de la Grèce antique concilie les approches textuelle et conceptuelle. Fruit d'une collaboration dans le domaine des objets matériels des cultures antiques initiée dès 2014, elle tient compte des expériences acquises lors de différents projets, dont deux projets européens dans le domaine du patrimoine, Linked Heritage et AthenaPlus. Cette méthodologie repose sur l'idée centrale qu'un concept est défini par un ensemble de caractéristiques suffisamment stable pour être désigné en langue par un terme. En effet, tout ensemble de caractéristiques compatibles entre elles ne définit pas nécessairement un concept. La langue joue donc un rôle important dans le "découpage " du monde en "figeant " par dénomination une connaissance du domaine et l'érigeant ainsi au rang de concept.

Cette méthodologie est dédiée à la construction d'ontoterminologies multilingues partageant une conceptualisation commune modélisée sous la forme d'une ontologie formelle. Elle repose sur une théorie du concept basée sur les notions de caractéristique essentielle et de carac- 
téristique descriptive, et pour laquelle les caractéristiques essentielles sont structurées en axes d'analyse ${ }^{41}$. C'est une méthodologie outillée à l'aide de l'environnement Tedi décrit au paragraphe précédent.

\section{Une méthode en cinq tâches}

La méthode Tedi est composée de cinq tâches liées entre elles, non nécessairement linéaires. Ces tâches sont illustrées avec l'exemple de la chlamyde ( chlamys » en anglais, « chlamyde " en français, « $\chi \lambda \alpha \mu u ́ s$ » en grec ancien et moderne) définie simplement comme un "short mantle, worn by horsemen " dans le dictionnaire LSJ. La figure 5 représente une chlamyde peinte sur vase. Notre objectif est la définition de quatre terminologies, en anglais, grec ancien, grec moderne et français, partageant la même dimension conceptuelle (ontologie).

FIGURE 5. LEKYTHOS

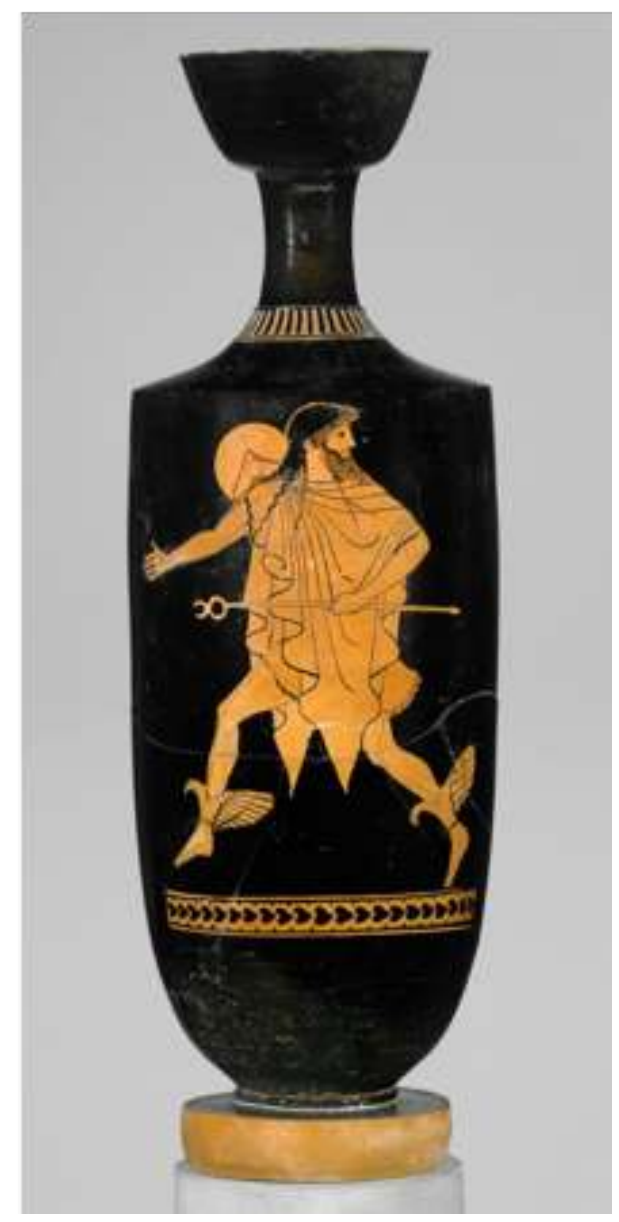

Lekythos (vase à huile) en terre cuite. Attribué au peintre de Tithonos. Époque classique (vers 480-470 avant notre ère). Culture : grecque (Attique). Matériau : terre cuite; vase à figures rouges.

New York : Metropolitan Museum of Art. Numéro de catalogue : 25.78.2. Fonds Fletcher, 1925. https://www.metmuseum.org/art/collection/search/251800. 


\section{Sélection des termes à définir (tâche 1)}

La première tâche consiste à sélectionner les termes à définir. Ces termes peuvent être extraits de corpus à l'aide d'extracteurs de termes candidats ${ }^{42}$ ou issus de méthodes de construction d'ontologies à partir de textes (Buitelaar et al. 2005). En ce qui nous concerne, les termes sont ici connus des experts : " chiton ", " chlamyde ", " himation ", " exomide ", "zeira ", etc. Les termes sont rentrés dans le système à l'aide de l'éditeur de termes (figure 7).

\section{Définition des caractéristiques essentielles et des axes d'analyse (tâche 2)}

En s'appuyant sur les connaissances des experts du domaine et sur les connaissances véhiculées par les différentes ressources (textuelles, visuelles, archéologiques), nous identifions un certain nombre de caractéristiques essentielles permettant de distinguer les différents types de vêtements référencés par les termes sélectionnés lors de l'étape précédente. Ainsi, on peut noter qu'une chlamyde, comme on peut le voir sur la figure 5, est portée attachée et non enroulée autour du corps comme l'himation, elle est faite d'une pièce contrairement au kandys, avec un seul point d'attache contrairement au chiton. Ces caractéristiques essentielles sont définies et structurées en axes d'analyse grâce à l'éditeur de concepts de Tedi. Ainsi l'axe d'analyse « body part » est défini par les caractéristiques essentielles exclusives entre elles /around body/, /around head/, /around waist/, /leg encasing/.

\section{Mise en relation des termes et des concepts (tâche 3)}

La troisième étape est au cœur de la démarche et implémente directement le principe sur lequel repose notre méthode. Elle consiste à lier termes et concepts (figure 6). Ainsi, pour chaque terme précédemment créé, on sélectionne l'ensemble des caractéristiques essentielles que l'on souhaite associer au terme. S'il n'existe pas de concept correspondant, l'environnement Tedi propose d'en créer un nouveau dont le nom est construit à partir des caractéristiques et dont la définition formelle est l'ensemble des caractéristiques qui ont été sélectionnées. Ainsi, en reprenant notre exemple, le terme "chlamys " en anglais dénote un ensemble de caractéristiques essentielles comprenant :/male/, /around body/, /one part/, /without sewing/, /one attachment/. Cet ensemble de caractéristiques constitue la définition formelle du concept dénoté par le terme " chlamys " et dont le nom proposé par Tedi est < Garment male around body one part without sewing... $>{ }^{43}$. Il suffit ensuite de préciser, que ce soit à l'aide de l'éditeur de termes de Tedi ou de l'éditeur de

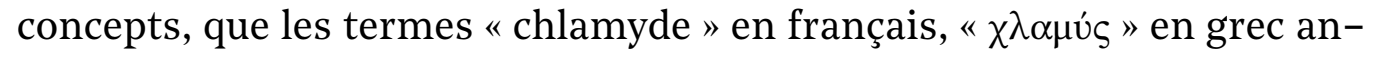
cien, " $\chi \lambda \alpha \mu u ́ s$ » en grec moderne dénotent le même concept. 


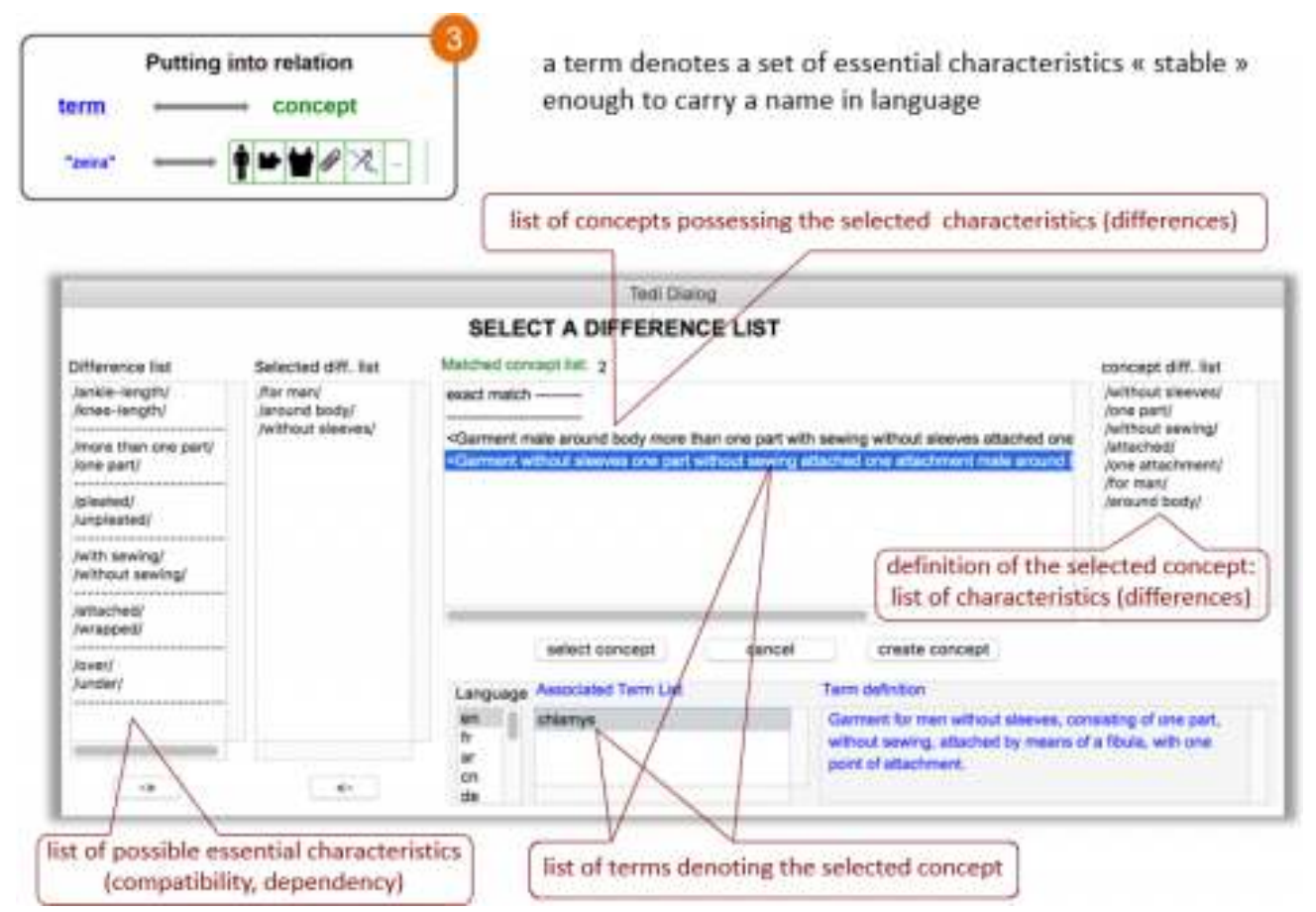

Schéma explicatif de l'interface du logiciel Tedi

La gestion des axes d'analyse, de leurs dépendances et de la compatibilité des caractéristiques essentielles est prise en charge par l'environnement Tedi. Elle permet également de guider l'expert dans la sélection des caractéristiques essentielles en ne proposant que celles qui sont à un moment donné possibles (figure 6).

\section{Dimension conceptuelle (tâche 4)}

Létape suivante porte sur la mise à jour de l'ontologie en insérant le concept nouvellement créé dans le système conceptuel. C'est-à-dire par sa mise en relation, hiérarchique ou associative, avec d'autres concepts, et en complétant, au besoin, sa description par l'ajout de caractéristiques descriptives. Le cas échéant, de nouveaux concepts peuvent être introduits à des fins d'organisation du système conceptuel sans qu'il existe pour autant de termes les désignant dans un système linguistique donné.

Afin d'aider à la structuration du système, Tedi indique automatiquement les concepts génériques possibles pour un concept donné (concepts génériques inférés), c'est-à-dire les concepts dont la définition intensionnelle (ensemble des caractéristiques essentielles) est incluse dans celle du concept en question. Par exemple, Tedi infère automatiquement que le concept dénoté par le terme "chlamys " compte $<$ Garment around body > et <Garment for man > parmi ses concepts génériques possibles qu'on peut alors déclarer explicitement comme tels afin d'en hériter les caractéristiques et de placer au bon endroit, pour ainsi dire, le concept dénoté par « chlamys » (figure 4). 


\section{Dimension linguistique (tâche 5)}

L'éditeur de termes de Tedi permet de compléter la définition des termes dans les différentes langues. À cette fin, Tedi propose des patterns de définition sur la base de la définition formelle du concept dénoté par le terme (qui en constitue la justification) et de son hyperonyme terminologique. Il reste à l'expert à les réécrire sous une forme syntaxique et stylistique plus correcte.

FIGURE 7. ÉDITEUR DE TERMES POUR « CHLAMYS »
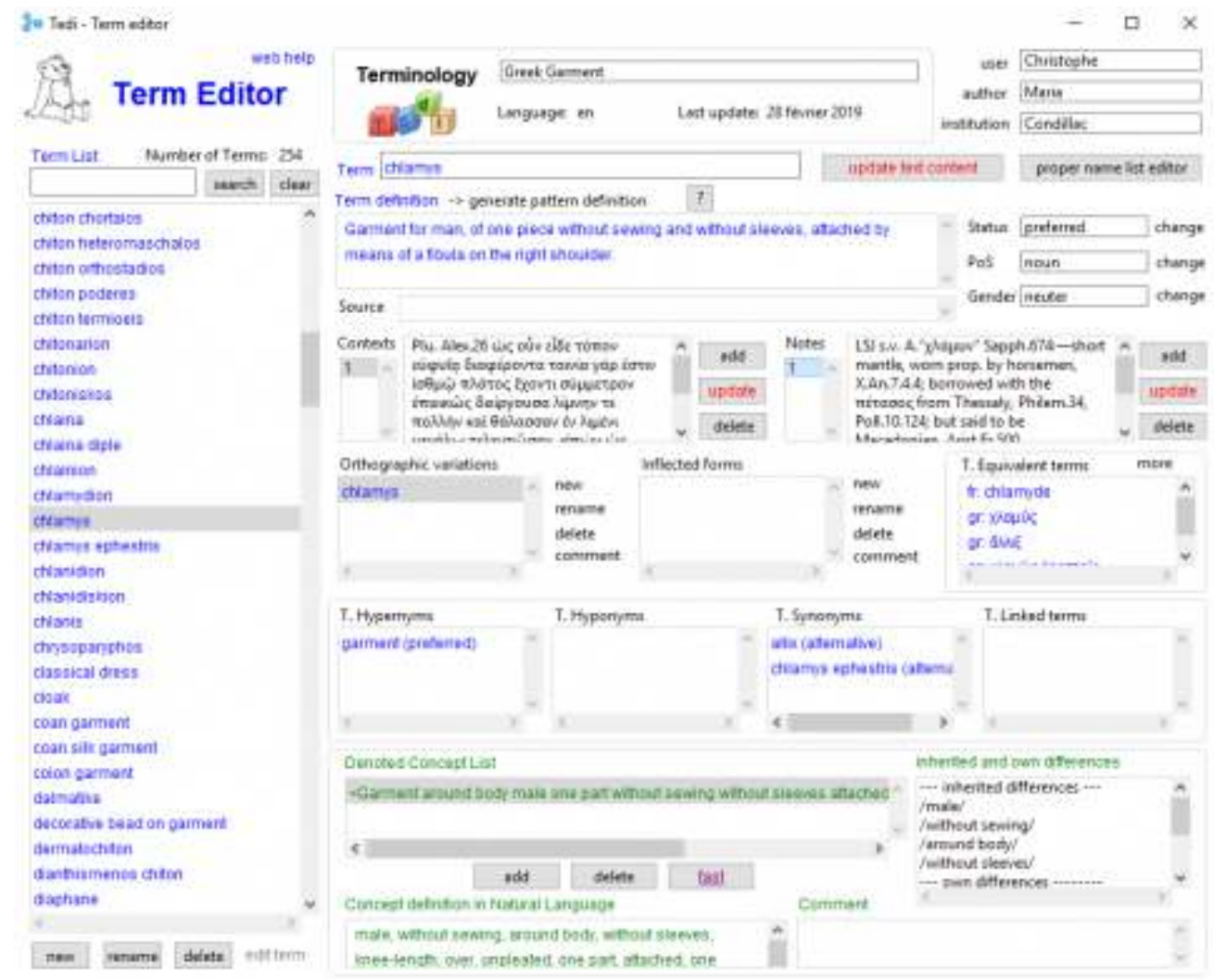

Capture d'écran du logiciel Tedi

En reprenant notre exemple, partant de la définition formelle du concept dénoté par le terme " chlamys ", nous l'avons défini de la façon suivante en anglais, français et grec moderne : «Garment for man, of one piece without sewing and without sleeves, attached by means of a fibula on the right shoulder", "Vêtement pour homme sans manche, constitué d'une seule pièce sans couture, il se porte attaché sur l'épaule droite à

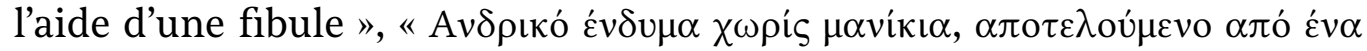

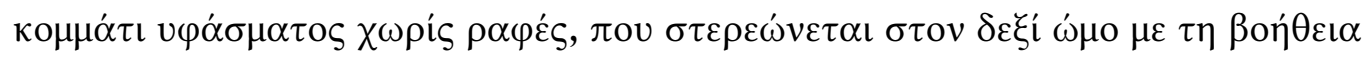

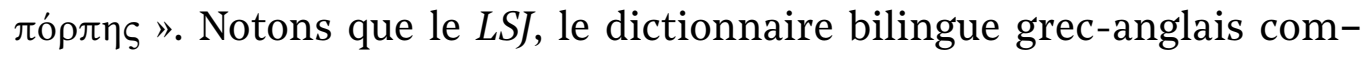
munément utilisé dans le domaine des études classiques, définit simplement la chlamyde comme un "short mantle, worn by horsemen". Modéliser les connaissances du domaine à l'aide de caractéristiques essentielles conduit à des définitions plus précises.

Les informations complémentaires, en particulier d'ordre encyclopédique portant sur la fonction du vêtement, sa composition (fibre), sa couleur, les vêtements associés, les personnes qui les portent, etc., ne font pas partie en tant que telles de la définition terminologique proprement dite du terme. Elles sont cependant cruciales pour la connaissance des vêtements de la Grèce antique et de la culture qui les a produits. Elles 
sont prises en compte par Tedi à travers les notes et les contextes (figures 7 et 9). Dans le cadre de cet article, nous présentons un nombre limité de contextes et de notes. Les informations relatives à la chlamyde dans la base de données OTB (OntoTerminology Database) des vêtements de la Grèce antique contiennent de nombreuses références et citations en lien avec les textes de référence du domaine ainsi que la description d'objets et d'images (figure 8).

FIGURE 8. L'ÉDITEUR D'OBJETS ET D'IMAGES DE TEDI

Jo Tedi - Object Editor

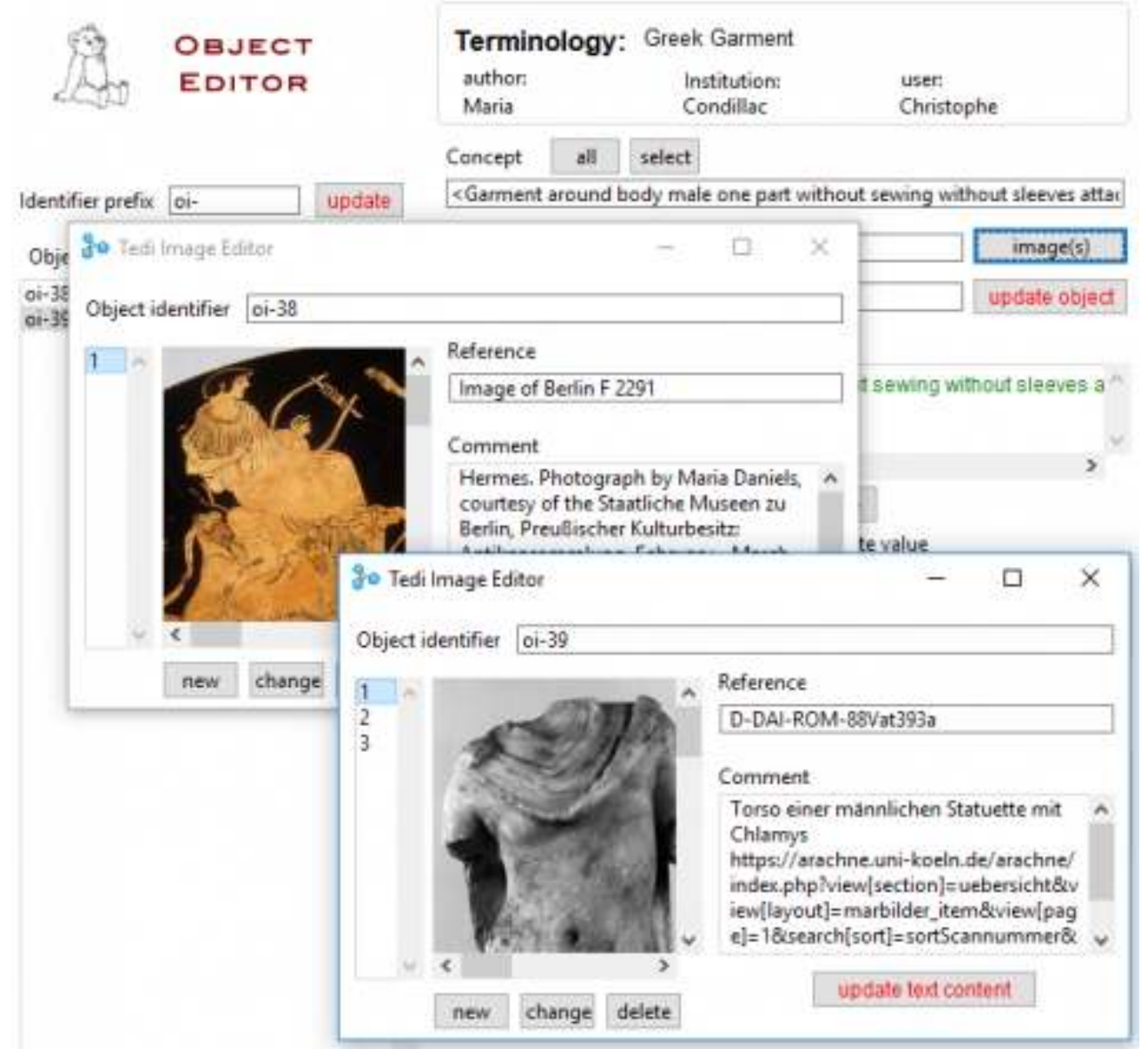

Capture d'écran du logiciel Tedi 


\section{Export des résultats}

La figure 9 présente l'entrée « chlamys » au format HTML ${ }^{44}$.

FIGURE 9. EXPORT DU TERME « CHLAMYS » AU FORMAT HTML

\section{Tedi Onto-Dictionary on "Greek Garment" (en)}

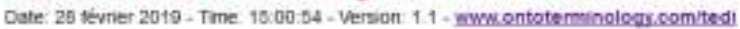

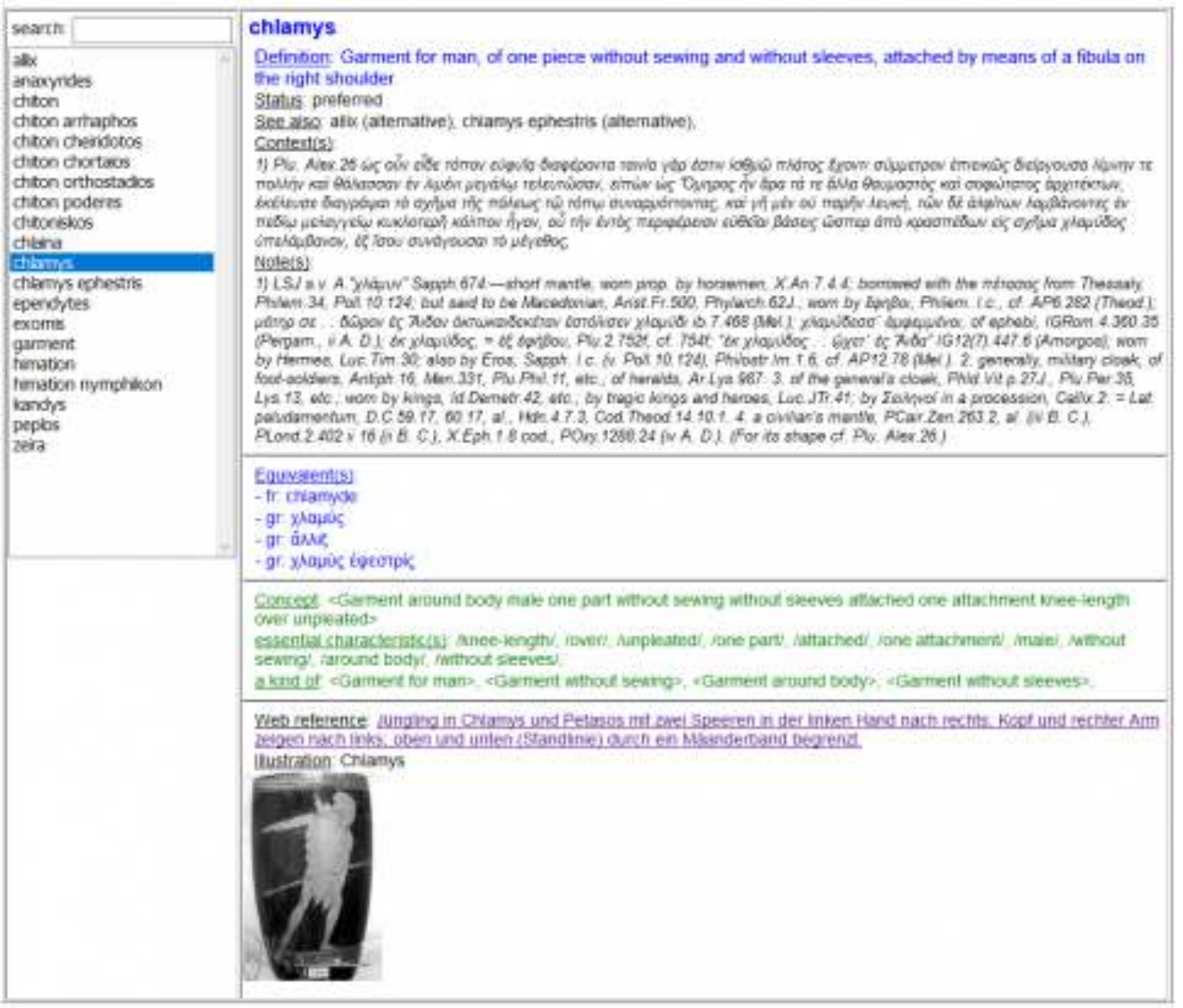

Capture d'écran du logiciel Tedi 


\section{De la nécessité d'une méthode outillée}

Les principes épistémologiques et les propriétés logiques de la théorie $\mathrm{du}$ concept sur lesquels repose l'ontoterminologie telle qu'elle est mise en œuvre dans Tedi sont "naturels " et facilement compréhensibles : caractéristiques essentielles (différences) et descriptives (attributs), relations (hiérarchiques, partitives, associatives), axes d'analyses regroupant des caractéristiques essentielles exclusives entre elles et portant sur un même sujet, dépendances entre caractéristiques essentielles, etc. Cependant, leur application n'est pas sans poser de problèmes lorsque la combinatoire devient trop importante. Ainsi, à partir de seulement dix axes d'analyse constitués de paires de caractéristiques essentielles opposées : (/avec couture/, /sans couture/), (/avec attaches/, /sans attache/), etc., il est possible de définir $1024\left(2^{10}\right)$ concepts terminaux. Si la dépendance entre caractéristiques permet de réduire ce nombre (par exemple, le nombre d'attaches n'a de sens que si le vêtement est porté attaché et non enroulé autour du corps), elle augmente la charge cognitive des experts qui doivent la prendre en compte lors de la conceptualisation. Un environnement informatique intégrant ces principes et qui en vérifie les propriétés logiques est une aide considérable d'un point de vue méthodologique en ne proposant à chaque étape que ce qui est autorisé au regard de ces propriétés et en indiquant ce qui peut être inféré : caractéristiques essentielles possibles, concepts génériques inférés, héritage de caractéristiques et d'attributs, mécanismes de recherche, etc.

\section{Conclusion}

Il ne peut y avoir communication, et a fortiori partage de connaissances, sans s'accorder sur les termes employés, c'est-à-dire sans s'accorder sur leur définition au regard des objets qu'ils désignent. La terminologie et la modélisation des connaissances du domaine sont au cœur de tout projet culturel. Si la notion d'ontologie, issue de l'intelligence artificielle, constitue ici une des voies les plus prometteuses (explicitation des connaissances du domaine, respect de la diversité linguistique, opérationnalisation des terminologies, etc.), elle n'est pas sans poser de problèmes, en particulier méthodologiques. L'existence d'environnements logiciels tels que l'éditeur d'ontologies Protégé constitue une aide importante, mais soulève d'autres questions d'ordre épistémologique, car un expert n'est pas obligatoirement familier avec les théories sous-jacentes à ces environnements (la logique des descriptions dans le cas de Protégé).

Ce projet a mis en évidence l'importance, voire la nécessité, de disposer d'une méthode outillée reposant sur des principes épistémologiques en accord avec le mode de pensée des experts. Nécessairement pluridisciplinaire, conciliant les démarches « émique » et «étique », puisant à la linguistique, la terminologie et la modélisation des connaissances, elle doit permettre la collaboration des différents intervenants et aboutir à des résultats partageables grâce à l'utilisation de formats d'échange respectant les standards en vigueur dont ceux de l'ISO et du $\mathrm{W}_{3} \mathrm{C}$. 
Nous avons ainsi abouti à une terminologie multilingue dont la signification des termes repose sur une ontologie du domaine des vêtements de la Grèce antique et qui peut donner lieu à différentes applications informatiques : dictionnaires terminologiques électroniques, moteurs de recherche sémantiques et multilingues, classification automatique, gestion documentaire, gestion des connaissances, etc.

Les vêtements de la Grèce antique ne sont pas les seuls objets matériels confrontés aux problèmes que nous venons d'énoncer. Notre approche, tant théorique que pratique, se veut applicable à d'autres domaines. Une étude est en cours sur la poterie de la Grèce antique.

\section{Bibliographie}

Baader, Franz, Diego Calvanese, Deborah McGuinness, Daniele Nardi et Peter Patel-Schneider. 2003. The Description Logic Handbook. Cambridge : Cambridge University Press.

Balfet, Hélène, Yvonne Broutin et Yves Delaporte. 1984. " Un essai de système descriptif du vêtement ". L'Ethnographie 92-94 : 363-373.

Berra, Aurélien. 2011. "Manier le thésaurus grec ». Dans Lieux de savoir. II. Les mains de l'intellect, édité par Christian Jacob, 555-578. Paris : Albin Michel. https://halshs.archives-o uvertes.fr/halshs-00556437.

Bettini, Maurizio. 2012. "Entre "émique" et "étique". Un exercice sur le Lar familiaris". Dans Anthropologie de l'Antiquité. Anciens objets, nouvelles approches, édité par Pascal Payen et Evelyn Scheid Tissinier, 173-200. Turnhout : Brepols.

Buck, Anne. 1982. Vocabulary of Basic Terms for Cataloguing Costume - Vocabulaire de base pour les fichiers de costume. Traduction française par Madeleine Delpierre. ICOM International Committee for the Museums and Collections of Costume. http://terminology.collectionstrust.org.uk/ICOM-costume/vbt00e.htm.

Buitelaar, Paul, Philipp Cimiano et Bernardo Magnini, éd. 2005. Ontology Learning from Text : Methods, Evaluation and Applications. Amsterdam : IOS Press.

Cleland, Liza, Glenys Davies et Lloyd Llewellyn-Jones. 2007. Greek and Roman Dress from A to Z. New York : Routledge.

Corcho, Oscar, Mariano Fernandez-Lopez et Asunción Gomez-Perez. 2003. « Methodologies, Tools and Languages for Building Ontologies. Where Is their Meeting Point ? ». Data and Knowledge Engineering $46:$ 41-64.

Crane, Gregory. 2017. « Design Sprint for Perseus 5.o. Open Greek and Latin ». Perseus Digital Library Updates. https://sites.tufts.edu/perseusupdates/2017/07/22/design-sprint-for-p erseus-5-0open-greek-and-latin/.

Crane, Gregory, Seals Brent et Melissa Terras, éd. 2009. "Changing the Center of Gravity : Transforming Classical Studies Through Cyberinfrastructure ». Digital Humanities Quarterly 3 (1). http://www.digitalhumanities.org/dhq/vol/3/1/.

Daremberg, Charles-Victor et Edmond Saglio, éd. 1877-1919. Dictionnaire des antiquités grecques et romaines. http://dagr.univ-tlse2.fr/.

Delaporte, Yves. 1981. « Pour une anthropologie du vêtement ». Dans Vêtement et sociétés, 1, Actes des journées de rencontre des 2 et 3 mars 1979, édité par Monique de Fontanès et Yves Delaporte, 3-13. Paris : Laboratoire d'ethnologie du Muséum national d'histoire naturelle et Société des amis du Musée de l'homme. https://halshs.archives-ouvertes.fr/halshs-000 04566.

Doerr, Martin. 2009. "Ontologies for Cultural Heritage ». Dans Handbook on Ontologies, édité par Steffen Staab et Rudi Studer, $2^{\mathrm{e}}$ éd., 463-481. Dordrecht : Springer. https://www.s pringer.com/gp/book/9783540709992.

Eicher, Joanne B. et Mary E. Roach-Higgins. 1992. « Definition and Classification of Dress : Implications for Analysis of Gender Roles ". Dans Dress and Gender : Making and Meaning, édité par Ruth Barnes et Joanne B. Eicher, 8-28. New York : Berg Publishers. https://conser vancy.umn.edu/handle/11299/170746.

Gherchanoc, Florence et Valérie Huet, éd. 2012. Vêtements antiques : s'habiller, se déshabiller dans les mondes anciens. Arles : Errance. 
Gras, Michel. 2000. «Donner du sens à l'objet. Archéologie, technologie culturelle et anthropologie ". Annales. Histoire, sciences sociales 55 (3) : 601-614. https://doi.org/10.3406/a hess.2000.279866.

Gruber, Thomas R. 1993. "A Translation Approach to Portable Ontology Specifications ». Knowledge Acquisition 5 (2) : 199-220.

Grüninger, Michael et Mark Fox. 1995. " Methodology for the Design and Evaluation of Ontologies ». Communication présentée au Workshop on Basic Ontological Issues in Knowledge Sharing, Montréal, Canada, 13 avril.

Guarino, Nicolas, Daniel Oberle et Steffen Staab. 2009. "What Is an Ontology ? ». Dans Handbook on Ontologies, édité par Steffen Staab et Rudi Studer, $2^{\mathrm{e}}$ éd. Dordrecht : Springer.

Harris, Marvin. 1976 (1968). « History and Significance of the Emic/Etic Distinction ». Annual Review of Anthropology 5 : 329-350.

Harris, Marvin. 1968. The Rise of Anthropological Theory. New York : Crowell.

Horridge, Matthew, Tania Tudorache, Jennifer Vendetti, Csongor Nyulas, Mark Musen et Natalya Noy. 2013. "Simplified OWL Ontology Editing for the Web : Is WebProtégé Enough ?». Dans The Semantic Web-ISWC 2013 Proceedings, Part I : 200-215. Springer.

ISO 1087-1. 2000. "Terminology Work - Vocabulary - Part 1 : Theory and Application ». Genève : International Standards Organisation.

ISO 25964-1. 2011. "Information and Documentation - Thesauri and Interoperability with Other Vocabularies - Part 1 : Thesauri for Information Retrieval ». Genève : International Standards Organisation.

ISO 25964-2. 2013. « Information and Documentation - Thesauri and Interoperability with Other Vocabularies - Part 2 : Interoperability with Other Vocabularies ». Genève : International Standards Organisation.

Liddell, Henry George, Robert Scott et Henry Stuart Jones. 1940. Greek-English Lexicon. $9^{\mathrm{e}}$ éd. Oxford : Clarendon Press.

Llewellyn-Jones, Lloyd. 2003. Aphrodite's Tortoise : The Veiled Woman of Ancient Greece. Swansea : Classical Press of Wales.

Losfeld, Georges. 1991. Essai sur le costume grec. Paris : Éditions de Boccard.

Pantelia, Maria. 200o. " "Noûs, into Chaos". The Creation of the Thesaurus of the Greek Language ». International Journal of Lexicography 13 (1) : 1-11.

Papadopoulou, Maria et Christophe Roche. 2018. "Ontologization of Terminology. A Worked Example from the Domain of Ancient Greek Dress ». AIDAinformazioni 36 (1-2) : 89-107.

Pike, Kenneth L. 2015 (1967). Language in Relation to a Unified Theory of the Structure of Human Behavior. Berlin : de Gruyter-Mouton.

Rastier, François. 2004. "Ontologie(s) ». Revue des sciences et technologies de l'information 18 (1) : 15-40. http://www.revue-texto.net/Inedits/Rastier/Rastier_Ontologies.html.

Rey, Alain. 1979. La Terminologie : noms et notions. Paris : Presses universitaires de France.

Roche, Christophe. 2005. «Terminologie et ontologie ». Langages 157 : 48-62.

Roche, Christophe. 2007. « Saying is Not Modelling ». Communication présentée au 4th International Workshop on Natural Language Processing and Cognitive Science : 47-56. Funchal, juin.

Roche, Christophe. 2012. "Ontoterminology : How to Unify Terminology and Ontology into a Single Paradigm ?». Communication présentée à la 8 th International Conference on Language Resources and Evaluation, 2626-2630, Istanbul, 21-27 mai.

Roche, Christophe, Luc Damas et Julien Roche. 2014. « Multilingual Thesaurus : The Ontoterminology Approach ». Communication présentée à l'International Committee for Documentation (CIDOC) : Access and Understanding - Networking in the Digital Era, Dresde, septembre.

Sardan (de), Jean-Pierre Olivier. 1998. «Émique ». L’Homme 147 : 151-166.

Staab, Steffen et Rudi Studer, éd. 2009. Handbook on Ontologies. $2^{\mathrm{e}}$ éd. Dordrecht : Springer. 
Stears, Karen E. 2006. "Dress and Textiles ». Dans The Edinburgh Companion to Ancient Greece and Rome, édité par Edward Bispham, Thomas Harrison et Brian A. Sparkes, 226230. Édimbourg : Edinburgh University Press.

Suárez-Figueroa, Mari Carmen, Asunción Gomez-Perez et Mariano Fernandez-Lopez. 2015. «The NeOn Methodology Framework : A Scenario-based Methodology for Ontology Development ». Applied Ontology 10 (2) : 107-145.

Uschold, Mike et Michael Gruninger. 1996. "Ontologies : Principles, Methods and Applications ». Knowledge Engineering Review 11 (2) : 93-136.

Uschold, Mike et Martin King. 1995. « Towards a Methodology for Building Ontologies ». Communication présentée au Workshop on Basic Ontological Issues in Knowledge Sharing, AIAI-TR-183, université d'Édimbourg, juillet.

Wielinga, Bob, Guus Schreiber, Jan Wielemaker et Jacobijn Sandberg. 2001. « From Thesaurus to Ontology ". Dans K-CAP '01, Proceedings of the 1st International Conference on Knowledge Capture, Victoria, Canada : 194-201.New York : ACM.

\section{Notes}

1 Pris au sens large en tant que " costume » : «on entend par costume les pièces de vêtement qui constituent par leur groupement fixe, la manière normale de se couvrir d'un groupe humain " (Leroi-Gourhan 1973), ce qui inclut d'autres formes de "modifications corporelles " telles que les parures, bijoux, tatouages, cosmétiques, coiffures, etc. Pour une brève présentation des équivalents anglais appearance, adornment, ornament, clothing, apparel, costume et fashion, voir Lee $(2015,21)$. Dans cet article, nous utiliserons " vêtement " au sens de «clothing or dress for the body".

2 Dans la Rome antique les sénateurs portaient la tunica laticlavi aux rayures violettes, les magistrats portaient la tunica angusticlavi, la toga palmata bordée d'or était destinée aux généraux tandis que les empereurs revêtaient une toge entièrement violette, la trabea.

3 Ce problème est récurrent dans de nombreux domaines, comme celui de la poterie : " Nous ne savons même pas, la plupart du temps, comment les Anciens nommaient les objets dont ils se servaient " (Gras 2000, 609).

4 La vaste bibliographie traitant de la nomenclature de l'habillement de la Grèce antique illustre la diversité des points de vue (par exemple Losfeld 1991 ; Llewellyn-Jones 2003 ; Cleland et al. 2007 ; Gherchanoc et Huet 2012 ; Lee 2015, pour n'en citer que quelques-uns), sans que pour autant émerge un consensus : «Rejetons, comme nous l'avons dit précédemment, une classification générale à la fois inutile et peu exacte que préconisèrent jadis certains auteurs, classification qui tendrait à séparer les vêtements grecs en deux catégories, ceux de dessous et de ceux de dessus " (Losfeld 1991, 90).

5 " Although the standard Greek and Latin terminology employed by scholars to describe ancient clothing may not be that which was used in antiquity to signify particular items of clothing, it is a useful vocabulary of dress. "

6 Introduits par Pike $(1967$; 2015) et fortement critiqués par Harris $(1968 ; 1976)$ les termes « émique " (emic) et "étique » (etic) dérivent respectivement de "phonémique " et "phonétique ». Pour une histoire de ces termes en opposition voir de Sardan (1998).

7 Même s'ils peuvent donner lieu à débats.

8 Une ontologie est une spécification (expression dans un langage compréhensible par un ordinateur) d'une conceptualisation d'un domaine, conceptualisation supposée partagée par une communauté de pratiques.

9 http://www.linkedheritage.eu/index.php.

$10 \mathrm{http}: / /$ www.athenaplus.eu.

11 «Despite the fact that automated classification is a long-established discipline of archaeology, there are no terminological systems that are widely accepted. "

12 Voir http://www.perseus.tufts.edu/ (Crane et al. 2009). Concernant la nouvelle version de Perseus, voir Crane (2017).

13 TLG: Thesaurus Linguae Graecae. A Digital Library of Greek Literature. Université de Californie à Irvine. http://stephanus.tlg.uci.edu. Voir Pantelia (2000) et Berra (2011, 6 et suiv.). 
15 https://www.beazley.ox.ac.uk/index.htm.

16 Citons pour exemple l'himation défini comme un "Dress in the form of a rectangular piece of cloth which may be worn over other dress, by women and old men, or on its own by men. It is usually draped over the left shoulder and across the waist, held in position by the left arm ". À cette définition sont associées deux représentations d'himation (poterie et statue). Voir https://www.beazley.ox.ac.uk/dictionary/Dict/ASP/dictionarybody.asp?name=Himation.

17 https://www.harvardartmuseums.org/collections.

18 https://arachne.uni-koeln.de/drupal/.

19 https://www.metmuseum.org.

20 https://www.europeana.eu/portal/fr.

21 Les mots de «vocabulaire ", « terminologie " et " thésaurus " sont dans cette discipline souvent employés indifféremment : «un thésaurus est un vocabulaire contrôlé structuré [...] ». L'emploi du mot " ontologie " est plus récent, directement lié à la part de plus en plus importante prise par le Web sémantique.

22 «[... thesauri and other types of vocabulary that are commonly used for information retrieval» (ISO 25964-2 2013).

23 Un thésaurus est défini comme un «controlled and structured vocabulary in which concepts are represented by terms, organized so that relationships between concepts are made explicit, and preferred terms are accompanied by lead-in entries for synonyms or quasisynonyms " (ISO 25964-1 2011).

24 Même si un thésaurus peut être traduit sous la forme d'une ontologie (Wielinga et al. 2001).

25 http://www.getty.edu/research/tools/vocabularies/aat/.

26 On distingue ici la terminologie dite conceptuelle où la définition des termes repose sur la définition des concepts - on parle alors de définition de chose - de la terminologie dite textuelle ou discursive qui s’intéresse aux sens (signifiés) des termes en discours. En cela, nous suivons la distinction faite par A. Rey entre définition terminologique et définition lexicographique (Rey 1979).

27 "The word "ontology" is used with different senses in different communities. The most radical difference is perhaps between the philosophical sense, which has of course a well-established tradition, and the computational sense, which emerged in the recent years in the knowledge engineering community, starting from an early informal definition of (computational) ontologies as "explicit specifications of conceptualizations". "(Guarino et al. 2009)

$28 \mathrm{Au}$ sens où ces connaissances sont définies dans un langage artificiel et non dans une langue naturelle.

29 Développé par le Centre de recherche en informatique biomédicale de l'université Stanford (Stanford Center for Biomedical Informatics Research, Stanford University School of Medicine), https://protege.stanford.edu.

30 «A Semantic Web language designed to represent rich and complex knowledge about things, groups of things, and relations between things " : https://www.w3.org/OWL/.

31 Tedi est développé par C. Roche à l'université Savoie-Mont-Blanc. Il peut être utilisé dans le cadre de projets de recherche : http://ontoterminology.com/tedi.

32 Il est à noter qu'un concept n’a pas nécessairement une désignation dans chaque langue.

33 Une caractéristique est essentielle si, retranchée de la chose, la chose n'est plus ce qu'elle est.

34 Afin de bien distinguer les deux dimensions, linguistique et conceptuelle, qui composent toute terminologie, nous utiliserons les conventions de notation suivantes : les termes sont notés entre guillemets et commencent par une minuscule, par exemple « himation " dénotant un type de vêtements particulier, les concepts entre chevrons commençant par une majuscule, par exemple < Vêtement pour homme sans manche> ; les caractéristiques essentielles sont notées entre barres obliques, par exemple /avec manches/ et /pour homme/ ; la référence à des objets est notée en italique, par exemple chiton, exomis ou chlamys.

35 Décrit l'état de la chose. Contrairement aux caractéristiques essentielles, les caractéristiques descriptives possèdent une valeur.

36 Même si dans certains cas la couleur est une caractéristique essentielle (voir la note 2). 
37 En indiquant sa signature, c'est-à-dire les types d'objets qui peuvent être liés par la relation, ainsi que ses propriétés (réflexive, symétrique, transitive, etc.).

38 Par exemple, dix questions suffisent pour sélectionner un concept terminal parmi $1024\left(2^{10}\right)$ concepts terminaux d'un arbre de Porphyre complètement développé sur la base de dix couples de différences opposées.

39 Pour une langue donnée, deux termes sont des synonymes terminologiques (à distinguer des synonymes discursifs communément appelés synonymes) si et seulement si ils dénotent le même concept. Le terme " $\mathrm{A}$ » est un hyperonyme terminologique (respectivement hyponyme terminologique) du terme « B » si le concept dénoté par " $\mathrm{A}$ » est un concept générique (respectivement concept spécifique) du concept dénoté par « $B$ ».

40 Il n'y a pas de concepts dans les textes, uniquement des traces de leur usage linguistique.

41 Les caractéristiques essentielles qui composent un axe d'analyse sont exclusives les unes des autres.

42 Voir par exemple la rubrique « Tools » dans les « Resources» du site https://termcoord.eu.

43 Si le terme est donné, le nom du concept est quant à lui construit de telle sorte qu'en le lisant on comprend la place du concept dans le système notionnel et la nature des objets qu'il subsume. La longueur du nom du concept n'est en rien un handicap, bien au contraire, dans la mesure où en discours ce sont les termes qui sont utilisés.

44 Un extrait de l'ontoterminologie des vêtements de la Grèce antique est accessible à l'adresse http://ontoterminology.com/e-dictionaries.

\section{Auteurs}

\section{Christophe Roche}

EA 3703 LISTIC, université Savoie-Mont-Blanc, Chambéry, France

Christophe Roche, issu du domaine de l'intelligence artificielle, est professeur à l'université Savoie-Mont-Blanc, responsable de l'équipe Condillac (Terminology \& Ontology) et de la recherche au laboratoire KETRC (Knowledge Engineering \& Terminology Research Centre, université de Liaocheng, Chine). Ses travaux portent sur la modélisation des connaissances (ontologies) et la terminologie.

christophe.roche@univ-savoie.fr

\section{Maria Papadopoulou}

EA 3703 LISTIC, université Savoie-Mont-Blanc, Chambéry, France

Maria Papadopoulou, philologue et linguiste, Marie Curie Fellow à l'université de Copenhague (2015-2017), est chercheuse associée à léquipe Condillac, responsable des humanités numériques au laboratoire KETRC (Knowledge Engineering \& Terminology Research Centre, université de Liaocheng, Chine). Ses travaux portent sur l'histoire du monde grec classique et hellénistique et la terminologie du vêtement de la Grèce antique.

maria.papadopoulou@univ-savoie.fr

\section{Droits d'auteur}

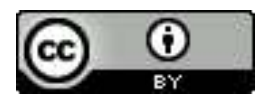

Les contenus de la revue Humanités numériques sont mis à disposition selon les termes de la Licence Creative Commons Attribution 4.0 International. 